\title{
Some Applications of Active Contours using ANN's*Isomorphic to Boundaries
}

\author{
Y.V. Venkatesh \& N. Rishikesh, \\ Computer Vision and Artificial Intelligence Laboratory, \\ Department of Electrical Engineering, \\ Indian Institute of Science, \\ Bangalore - 560 012,India.
}

\begin{abstract}
We present a few applications of the neural active contour model described in [1]. The model, which is a neural network based on the principles of self-organization, exploits the principles of spatial isomorphism and self-organization in order to create flexible contours for characterizing shapes in images. In this paper, we illustrate the versatility of the model by presenting its applications to the problems of boundary extraction, stereo vision, bio-medical image analysis and digital image libraries. For lack of space, only the essential ideas are described here. For details, see [1].
\end{abstract}

\section{Introduction}

Computational vision deals with the problem of extracting the shapes of two- and three-dimensional objects from images of physical scenes. Most of the present literature deals with model-based techniques which use a model of the object whose boundary representation is matched to the image in order to extract the boundaries of the object. The models used in such a process could be either rigid, as in the case of simple templatematching approaches, or nonrigid, as in the case of deformable models. The latter, which deform themselves in the process of matching, have come to be known as Active $^{1}$ Contour Models (ACM). By virtue of their greater flexibility than the earlier rigid models, they have been effectively employed in resolving various problems in vision: stereo matching [2], motion tracking [2], detection of subjective contours[2], segmenting biomedical images [12], face recognition [3], and so on.

The paper is organized as follows: Sec. 2 illustrates the need for Active Contour Models in the area of boundary extraction, by showing the inabilities of the

\footnotetext{
*Artificial Neural Networks

${ }^{1}$ to represent the dynamical nature of the models in the process of matching
}

previous methods in extracting boundaries and by showing the advantages of ACM's over them. Sec. 3 describes the existing ACM's and the various approaches used towards achieving the goal of modelling contours. Sec. 4 describes, in brief, the active contour model proposed in [1], along with a description of the constraints imposed and the various methods of initialization. Sec. 5 describes the applications of the model to contour extraction, stereo-image analysis, biomedical image interpretation, and image libraries. Sec. 6 concludes the paper.

\section{Need for ACM's}

Boundary detection along with the associated operation of edge linking is a difficult and challenging problem in image analysis. The set of pixels obtained from the edge detection algorithms seldom characterize a boundary completely because of noise, breaks in the boundary as a result of non-uniform illumination, and other effects that introduce spurious intensity discontinuities[6]. Before the advent of ACM's, the process of edge linking was to be undertaken after the edge detection algorithm in order to obtain a useful boundary representation. For instance, the characteristics of edge pixels are analyzed in a small neighborhood about every point in the image. All the points that are similar (in some sense) are then linked, thereby forming a boundary of pixels that share common properties. Other techniques used are: graph-theoretic [6], which cannot be used for realtime processing; and Hough transform-based [7], which involves only global shape information without taking into account local variations, and hence belongs to the category of rigid templates. In brief, the earlier shape detection algorithms have rigid templates, without allowing for any deformation. This makes them virtually unfit for applications involving distortion or variation in shape, non-rigid motion tracking, and the like. 
In an attempt to avoid these disadvantages, a deformation strategy was introduced into the templates (boundary representations), thereby leading to the ACM's. These models use higher level image interpretation techniques for placing the initial global contour, and later employ local (and/or global) deformations in order to achieve a match between the model and the object boundary. Further, as they can deform themselves in the process of matching the boundaries, they are well suited for applications which require handling distortions. Moreover, these models effectively use information from higher level image interpretation algorithms to deal with early visual processing, conceived to be a heterarchical model of the vision system rather than the hierarchical model. This turns out to be expedient, because a rigidly sequential bottom-up approach propagates errors made at a low level to higher levels, without any possibility for correction [2].

\section{Existing Models}

The snake [2] is probably the first proposed ACM, which is a controlled continuity-spline under the influence of internal (spline), image and external (constraint) forces. The internal spline forces impose a smoothness constraint, while the image forces push the snake towards salient features (lines, edges, subjective contours, etc.). The external constraint forces, on the other hand, are responsible for placing the snake near the desired local minimum, and originate from the choice of the initial contour, which, in turn, is governed by higher level image interpretation algorithms. The problem of contour modelling is then cast in the framework of energy minimization, with the energy functions consisting of terms corresponding to the internal, image and external forces. In the attempt to overcome some of the shortcomings of the snake model, the ACM's proposed in the literature either modify the energy functionals used in the original snake model or propose new approaches. See $[8,9]$ and also [10].

\section{Neural Active Contour Model}

In [1], we have proposed a new active contour model based on self-organization. This model, which differs completely from the other models (in both the underlying theory and its implementation), is a modification of the neural-network model, described in [4]. For an application of this idea to the highly successful recognition of 2-D objects and characters (subject to rotation and scaling), see [4]. The active contour model proposed by in [1] uses a self-organization scheme similar to Koho- nen's [11] algorithm, but is completely different from it in terms of architecture. In fact, the method does not employ the neural architecture with a lattice of neurons, typical of Kohonen's network.

The present model, as with most of the present ACM's, requires an initial contour, starting from which it evolves. A neural network isomorphic to this initial contour is constructed, and subjected to deformation in order to map onto the nearest salient contour in the image. The correspondence between the salient contour and the network is established by mapping the latter onto the former by using the self-organization scheme $[11,5]$. See [1] for a description of the various steps involved.

The model employs two major constraints which implicitly impose the smoothness constraint. The internal and image forces are, respectively, introduced by the constraints given below, and by the input fed to the network. As far as the external constraint force is concerned, it is made implicit by virtue of the fact that the initial contour is provided by higher level interpretation processes (or a user interface). The two constraints employed in the contour model are those on (i) the WinnerMaximum-Distance (WMD), and (ii) the Neighborhood Constraint Parameter (NCP). The WMD constraint is useful in avoiding the influence of edge points which are within the region of interest, but are not a part of the nearest salient contour of interest (spurious edge points). In the absence of such a constraint, the neurons "learn" spurious edges, thereby affecting the proper extraction of the desired contour. The NCP refers to the maximum of the distances in the $x-$ and $y$ - directions, taken over all the adjacent pairs of points on the contour. Constraining this parameter helps in maintaining the continuity of the contour in the course of its deformation. In the absence of a constraint on this parameter, many neurons tend to learn a single point of the input image, leading to discontinuities in the final contour.

The proposed method, being a semi-automatic image analysis technique, requires a rough boundary as the initial contour to start the deformation process. This initialization can be achieved in a number of ways, depending on the application. For static scenes, the generalized Hough transform [7] technique (see [8]) can be employed to initialize the contour, thereby exploiting the efficiency and globality of the Hough transform in the presence of noise and gaps in boundary. On the other hand, in an active vision system with movable (and multiple) cameras, two or more images could be acquired, and subjected to optical flow analysis or image differencing techniques, the results of which could be used to initialize the contour. 


\section{Applications of the model}

In order to illustrate the versatility of the model, we apply it to the following problems of vision: boundary localization and extraction; stereo image analysis, bio-medical image processing, and digital image library creation. The implementation of the algorithms has been done in $C++$ language with $X 11$ for graphical user-interface. The experiments are conducted on a HP9000/715 workstation. For a description of the various characteristics of the model, the values of parameters employed, and the advantages of the model with respect to other models, see [1].

\section{Boundary Extraction}

The model is applied to the problem of localization and extraction of boundaries in the course of segmenting image data. An example to illustrate this aspect is shown in Fig. 1. Fig. 1(a) shows the image overlaid with the initial contour. Fig 1(b) shows the edge image corresponding to the image under consideration, which clearly shows the internal spurious edges. Fig. 1 (c) shows the the final contour overlaid over the image, thereby illustrating the ability of the approach in extracting boundaries.

\section{Stereo Vision}

The proposed ACM provides a novel technique to efficiently extract and match, point-by-point (for disparity estimates), the corresponding contours from the left and right images of a stereo-pair of images. We outline below the steps involved in solving this correspondence problem. Fig. 2(a) shows a stereo-pair of images which are to be matched for depth extraction.

1. Extract contours from both the left and right images of the stereo pair. (We call them left and right contours, respectively.)

2. Form a neural network isomorphic to either the left or the right contour. That is, form a network, with weights of the neurons set to the co-ordinates of the contour points. Without loss of generality, we assume that the network is constructed isomorphic to the left contour.

3. Present each point from the right contour to each neuron in the network, and use the updating scheme described for contour extraction given in [1]. Dispense with the WMD and NCP constraints.

4. When the network converges, it is equivalent to the contour extracted from the right contour. The initial and final weights of a particular neuron will be the corresponding contour points in the left and right images, respectively. Also, assuming epipolar constraint, the difference between the $X$ coordinates of the initial and final weights of any particular neuron gives the disparity at that point (which can be used to calculate the depth of the point).

The disparity map is shown in Fig. 2(b) in which intensity is directly proportional to the disparity (and inversely proportional to the depth). In the example shown, the disparity map for the cube was obtained by separately initializing contours for the three surfaces (see Fig. 2(a)), and calculating the disparity for each of them, and, finally, merging them together.

\section{Biomedical Image Analysis}

Imaging techniques like Magnetic Resonance Imaging(MRI), X-ray Computed Tomography(CT) and Positron Emmision Tomography (PET) provide detailed information regarding the anatomical and physiological functions of the various parts of the body. However, an interpretation of the data has been hindered by the inability to relate such information with specific anatomical regions. This is a consequence of the difficulties that arise due to small variations in anatomy [12]. Because the earlier models for shape are rigid, it is not possible to accomodate these variations for better interpretation. This can be overcome by employing active contour models, which deform themselves in the process of extracting the boundaries. Furthermore, medical applications (like cardiac boundary tracking, tumor volume quantification, cell tracking ete.,) require extracting exact shapes in two and three dimensions. These also have been challenging tasks because of the amount of noise inherent in medical images. Active contour models can be used to extract boundary descriptions which are implicit in such noisy images.

Now we illustrate the application of our model in the extraction of implicit boundaries from bio-medical images, thereby facilitating an easy interpretation of anatomical parts. Fig. 3(a) shows an ultra-sound image of the head, overlaid with the initial contour. Fig. 3(b) illustrates the ability of the approach in extracting the contor information implicitly present in the image.

\section{Object Retrieval and Image Libraries}

The proposed model can be considered as a deformable template for application to the problem of locating and retrieving an object from a complex image. A solution to this problem is of significance to applications, like image database retrieval, object recognition and image segmentation. The proposed approach can 
be employed in a fashion similar to the one reported in [10]. In this context, it is assumed that a priori information is available in the form of an inexact model of the object, which needs to be matched with the object in the input image.

Since the proposed contour model gives a continous set of points as output after the deformation and matching, it is possible to use the model as a deformable template in object matching applications. The weights of the model weights are initialized with the co-ordinates of the binary template, and deformation is done in much the same way as described in [1]. The only difference lies in the search of the parameter space corresponding to scale, shift and rotation of the pattern, with the model initialized by the transformed versions of the binary template. However, the disadvantage of using such an approach (as with that of [10]) is the amount of time required in searching the entire parameter space. This can be reduced by the use of a coarse-to-fine matching strategy [10]. However, the parameter space dimension of the method proposed in [10] is high because the deformations are also considered as parametric functions. In contrast, the proposed approach reduces the dimension of the parameter space to four, corresponding to rotation, scale and shifts in $\mathrm{X}$ - and $\mathrm{Y}$-directions. This is a direct consequence of the fact that the deformation is handled by the active contour model itself.

\section{Conclusions}

We have demonstrated the versatility of the neural active contour model proposed in [1], by illustrating its application to typical problems in computational vision. In the course of locating the desired contour in a given image, a contour, chosen initially to be the given contour itself, is made to undergo deformation by a locally co-operative, but globally competitive scheme. This deformation enables the initial contour to cling to the nearest salient contour in the image. Examples elucidate the primal contour matching strategy for resolving problems in stereo vision, biomedical image analysis and digital libraries.

\section{References}

[1] Y.V. Venkatesh and N. Rishikesh, "Modelling Active Contours using Neural Networks Isomorphic to Boundaries," Presented at International Conference on Neural Networks(ICNN-97), Houston, Texas, USA, June 1997.
[2] M. Kass, A. Witkin, and D. Terzopoulos, "Snakes: Active contour models", Proc. First IEEE Conference on Computer Vision, pp. 259-268, 1987.

[3] A.L. Yuille, P.W. Hallinan and D.S. Cohen,"Feature Extraction from faces using deformable templates," International Journal of Computer Vision, Vol. 8, No. 2, pp. 133-144, 1992.

[4] C.N.S. Ganesh Murthy and Y.V. Venkatesh, "Applications of Self-Organization Networks Isomorphic to Patterns", Submitted to IEE Proceedings, Vision, Image and Signal Processing(UK).

[5] C.N.S. Ganesh Murthy, K. Shanmukh and Y.V. Venkatesh, "A New Method for Pattern Recognition Using Self-Organizing Networks", Proc. Second Asian Conference on Computer Vision(ACCV), Singapore, Vol.3, pp. 111-115, 1995.

[6] R.C. Gonzalez and Richard E. Woods, Digital Image Processing, Addison Wesley, 1992.

[7] D.H. Ballard, "Generalizing the Hough Transform to detect arbitrary shapes", Pattern Recognition, Vol 13(2), pp. 111-122, 1981.

[8] K.F. Lai and R.T. Chin, "Deformable contours : Modelling and Extraction", IEEE Pattern Analysis and Machine Intelligence, Vol. 17, no 11, pp. 1084 $-1090,1995$.

[9] Laurent D. Cohen, "On active contours models and balloons," Computer Vision, Graphics and Image Processing : Image Understanding, Vol. 53, No. 2, pp. 211-218, Mar. 1991.

[10] Anil K. Jain, Yu Zhong and Sridhar Lakshmanan, "Object Matching Using Deformable Templates," IEEE Transaction on Pattern Analysis and Machine Intelligence, Vol. 18, No. 3, March 1996.

[11] T. Kohonen, Self Organization and Associative Memory, Springer-Verlag, 1989.

[12] M.I. Miller, G.E. Christensen, Y. Amit, U. Grenander, "Mathematical Textbook of Deformable Neuroanatomies," Proceedings of the National Academy of Sciences, USA, Vol. 90, pp. 1194411948, Dec. 1993. 


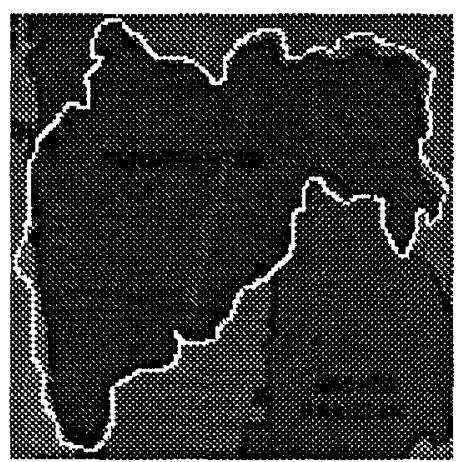

(a)

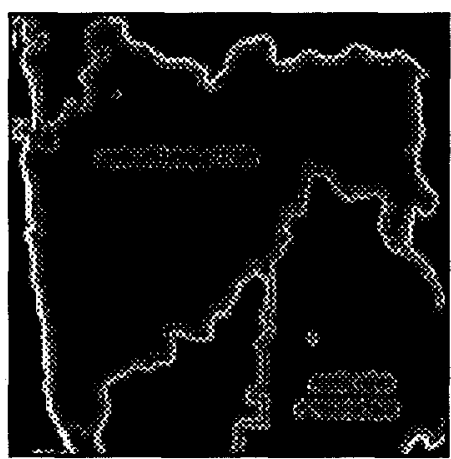

(b)

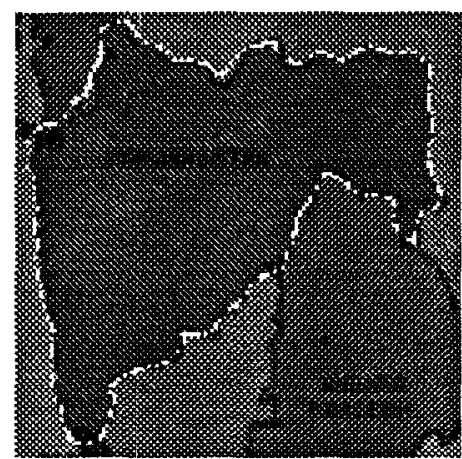

(c)

Fig. 1: Application of the model to extraction of boundaries

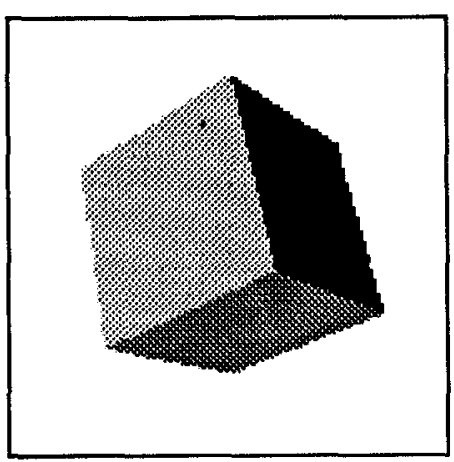

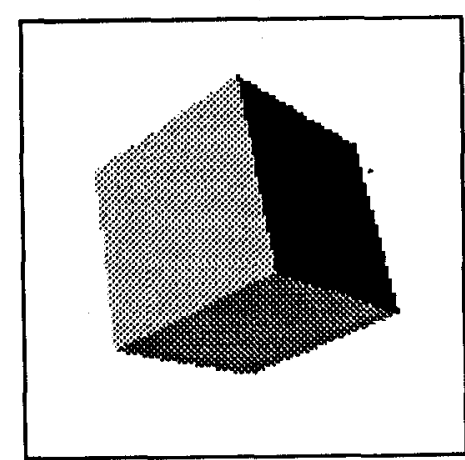

(a)

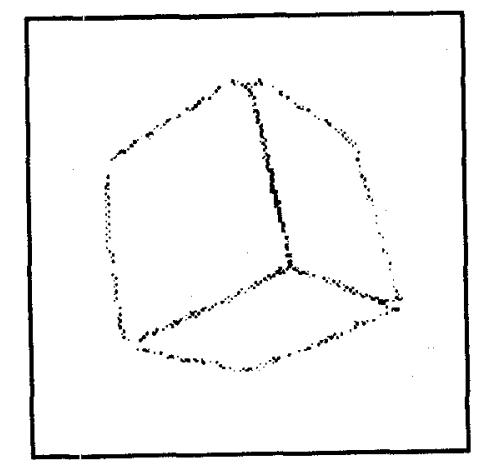

(b)

Fig. 2: Application of the model to stereo image analysis

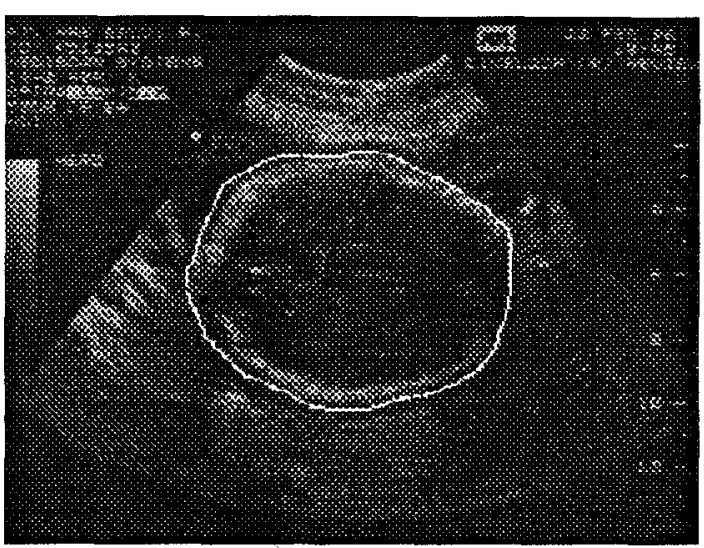

(a)

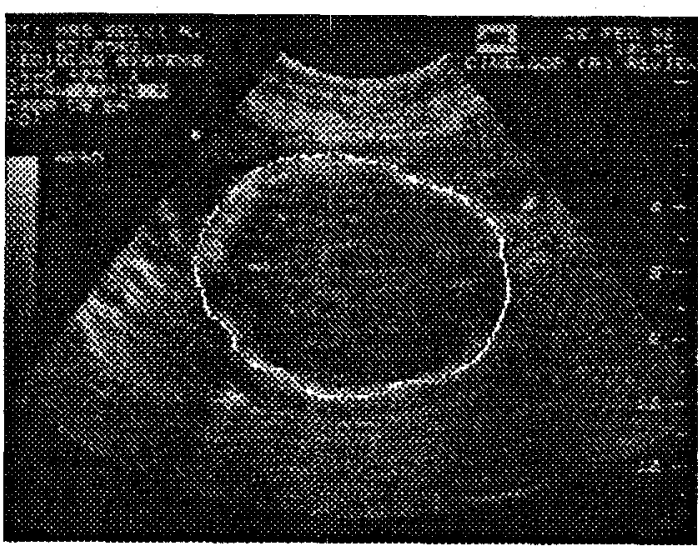

(b)

Fig. 3: Application of the model to bio-medical image analysis 